\title{
Lebenslange Lerner - Bildungsbiografische (Dis-)Kontinuitäten differenter Formen des Lernens von Fremdsprachen
}

\author{
Sigrid Nolda $\cdot$ Jochen Kade
}

Online publiziert: 26. März 2015

(C) Die Autor(en) 2015. Dieser Artikel ist auf Springerlink.com mit Open Access verfügbar

Zusammenfassung In diesem Artikel wird am Beispiel des Fremdsprachenlernens der wissenschaftlich noch wenig bearbeitete Zusammenhang zwischen Identität und Lernen thematisiert. Annahme ist, dass trotz des bildungspolitischen Appells zum Lebenslangen Lernen das Lernen im Lebenslauf nicht kontinuierlich, sondern diskontinuierlich ist. Es wird die Frage gestellt, wie die Lernenden mit diesen Diskontinuitäten umgehen. Die Wahrnehmung, Beschreibung und Ordnung dieser Diskontinuitäten ist - so die These des Beitrags - abhängig vom Selbstbild als Lerner bzw. von dessen Identität. Drei Formen werden empirisch herausgearbeitet: die Identität als heroische(r) Lerner(in), die Identität als selbstverständliche(r) Lerner(in) und die Identität als potenzielle(r) Lerner(in). In allen drei Formen, die keineswegs ausschließend sind, sondern sich individuell im Lebenslauf abwechseln können und zugleich zeitbezogen unterschiedliche Verbindungen eingehen können, wird Kontinuität als lebenslanger Lerner her- bzw. dargestellt; und dies auch dann, wenn das Lernen diskontinuierlich verläuft.

\begin{abstract}
The following article focuses the neglected relation between identity and learning by reconstructing foreign language learning efforts by adults. In spite of educational policy's proclamation of (continuous) lifelong learning, real learning processes are characterised by various discontinuities. Adults deal with these discontinuities by perceiving, describing and structuring them dependent on their selfimages as learners. Interpretations of interviews with adult language learners supply
\end{abstract}

\footnotetext{
Univ.-Prof. Dr. S. Nolda ( $\bowtie)$

Technische Universität Dortmund,

Dortmund, Deutschland

E-Mail: Sigrid.Nolda@fk12.tu-dortmund.de

Prof. Dr. J. Kade

Goethe-Universität Frankfurt am Main,

Frankfurt am Main, Deutschland

E-Mail:kade@em.uni-frankfurt.de
} 
evidence for three strategic forms of identity: the identity as a "heroic" learner; the identity as a "natural" learner; and the identity as a "potential" learner. The different identities, altering during the course of life, maintain continuity even when learning activities turn out to be discontinuous.

\section{Diskontinuitätserfahrungen und Kontinuitätserwartungen des Lebenslangen (Fremdsprachen-)Lernens}

Der nicht nur von der erziehungswissenschaftlichen Forschung zum Lebenslangen Lernen thematisierten, sondern auch von der Politik, Soziologie und Lebenslaufforschung zum Thema gemachten Diskontinuität moderner Lebensläufe und Biografien (Kade und Seitter 1996; Kade und Nolda 2012b, 2014a, b; Hof et al. 2010; zu Erwerbsbiografien Behringer et al. 2004), wird häufig - direkt oder indirekt - die Kontinuität des Lebenslangen Lernens entgegengehalten: als praktische Strategie der Bewältigung gesellschaftlicher Anforderungen oder aber als Möglichkeit der Selbstfindung. Aber die Kontinuität, die der Begriff des Lebenslangen Lernens und deutlicher noch der der éducation permanente suggeriert, ${ }^{1}$ wird dem wechselvollen Ablauf des Lernens Erwachsener, dem Aussetzen, Aufgeben oder Wiederaufgreifen, dem Umwidmen, Vermischen und Auslaufen einzelner Lernaktivitäten nicht gerecht. Nimmt man das Lernen über die gesamte Lebensspanne in den Blick, dann treten eher Diskontinuitäten hervor: der Lerngegenstände, der Lernformen, der Lernmedien, der Lernumgebungen usw. ${ }^{2}$ Gleichwohl wird mit dem bildungspolitischen Appell zum Lebenslangen Lernen eine Kontinuität des Nicht- oder nur bedingt Kontinuierlichen propagiert. Dieser legt den Individuen nahe, Diskontinuitäten zu vermeiden bzw. die von ihnen erlebten oder auch herbeigeführten Diskontinuitäten umzuinterpretieren und als Bestandteile einer übergeordneten Kontinuität wahrzunehmen bzw. darzustellen. Der folgende Beitrag verdeutlicht, dass man die Spanne zwischen Kontinuitätsanforderungen und Diskontinuitätserfahrungen als Verhältnis zwischen eher stabilen Identitätskonstruktionen von Lernern einerseits und dem Umgang mit Erfahrungen von realer Diskontinuität andererseits beschreiben kann. Es wird die These vertreten, dass die narrativen Lernidentitäten die erfahrenen bzw. erzählten Diskontinuitäten überformen.

Exponierte soziale Situationen, die zur Verbalisierung dieser (Um-)Interpretation auffordern, sind biografische Interviews, die sich direkt oder indirekt auf die Lern- und Bildungsvorgänge der Befragten richten. Der Aufforderung, Lernaktivitäten oder auch bildungsrelevante Erlebnisse der Vergangenheit und Gegenwart zu schildern und einen Ausblick auf die Zukunft zu geben, kann kaum entsprochen werden, ohne zugleich Zusammenhänge herzustellen. Dem Zwang zur Darstellung von Identitäts-Kohärenz trotz bestehender Inkohärenzen in biografischen Erzählungen (Lucius-Höhne und Deppermann 2004) entspricht der Zwang zur Darstellung

\footnotetext{
${ }^{1}$ Vgl. die grundlegende Analyse der Programmatik des Lebenslangen Lernens im Prozess seiner bildungspolitischen Institutionalisierung von Rausch 2014.

${ }^{2}$ Vgl. auch Kade und Seitter 1996 am Fall der Modi der Aneignung des Funkkollegs im Kontext des Lebenslangen Lernens.
} 
von permanentem Lern-Interesse trotz bestehender Diskontinuitäten realer und vor allem nachweisbarer Lernaktivitäten. So wie das erzählende Ich trotz - notwendiger - Änderungen und Wandlungen das gleiche bleiben muss, so müssen auch seine Lernaktivitäten trotz der - unvermeidlichen - Brüche in einem (Bereitschafts-)Kontinuum dargestellt werden, in dem es immer wieder zu Lernaufnahmen und Lernfortsetzungen kommt und das auch in der Rückschau konkrete Diskontinuitäten in einem allgemeinen Kontinuum verortet. Die Analyse biografischer Interviews informiert daher nicht nur über die Diskontinuitäten, die die Befragten erfahren haben, sondern - bei näherer Betrachtung - auch darüber, wie sie diese objektiven und subjektiv erfahrenen Diskontinuitäten darstellen und praktisch damit umgegangen sind.

Was für den bildungsbiografischen Umgang mit Diskontinuitäten allgemein gilt, trifft auch auf den Umgang mit Diskontinuitäten von einzelnen Lernpraktiken zu. ${ }^{3}$ Praktiken sind somit weniger individuell als sozial, weniger intentional als habituell, sie sind auch dann virulent, wenn sie (gerade) nicht explizit ausgeführt werden. Sie stehen gewissermaßen permanent zur Verfügung. Dies trifft auch für das Lernen fremder Sprachen zu (Nolda 2014), wenngleich diese Lernpraktik bei bildungsnahen Erwachsenen zweifellos deutlicher in Erscheinung tritt und bei ihnen auch eher und vielfältiger realisiert wird. ${ }^{4}$

Die Sprachlern(erwerbs)forschung hat zusammen mit der Lernpsychologie darauf hingewiesen, dass erwachsene Lerner (nicht nur Lernerinnen) durchaus fähig sind, Sprachen bis zu einem hohen Kompetenzniveau zu lernen, auch wenn sie in Bereichen Aussprache und Morphosyntax Jüngeren unterlegen sind (Grotjahn et al. 2010). Nicht die Lernfähigkeit ist das Problem, sondern die Aufrechterhaltung des Lernens bzw. einer Lernhaltung über einen möglichst langen Zeitraum hinweg - ungeachtet von Veränderungen der besuchten Institutionen, der verwendeten Lehr-/Lernmedien, der involvierten Personen usw.

Die gesellschaftliche Erwartung an Erwachsene, sie müssten in mindestens einer Fremdsprache kommunizieren können, muss nicht unbedingt mit dem Besuch von entsprechenden Unterrichtsveranstaltungen assoziiert werden. Dies macht die mittlerweile erfolgte Aufwertung selbstorganisierten und informellen Lernens deutlich. Dieser „Freiheit“ steht der ,Zwang“ entgegen, in Bewerbungslebensläufen oder Portfolios die solcherart erworbenen Kenntnisse zu dokumentieren und zu evaluieren etwa mit der Angabe der Kompetenzstufe, wie sie vom Gemeinsamen Europäischen Referenzrahmen für Sprachen definiert wird (www.europaeischer-referenzrahmen. de).

Mit dem in der Regel curricularisierten Sprachenlernen ist die Idee eines Anfangs und eines Endes verbunden. Das ist augenfällig in Abfolgen wie denen von Anfän-

\footnotetext{
${ }^{3}$ Nach Reckwitz enthalten soziale Praktiken ,in sich Handlungsakte, die wiederholt hervorgebracht werden, aber während das Konzept der ,Handlung' sich punktuell auf einen einzigen Akt bezieht, der als intentionales Produkt eines Handelnden gedacht wird, ist eine Praktik von vornherein sozial und kulturell, eine geregelte, typisierte, von Kriterien angeleitete Aktivität, die von verschiedensten Subjekten getragen wird“" (Reckwitz 2003, S. 38).

${ }^{4}$ So ist das Erlernen des Italienischen und Französischen häufig mit den spezifischen kulturellen Interessen und touristischen Aktivitäten der Mittelschicht verbunden. Spätestens mit der Einführung des Englischunterrichts an Hauptschulen ist Englisch die am wenigsten exklusive Fremdsprache und gehört zum allgemeinen Bildungsfundus.
} 
ger-, Fortgeschrittenen- und Konversationskurs. Die Tatsache, dass mehr Anfängerals Fortgeschrittenenkurse angeboten werden, weist darauf hin, dass Sprachkurse häufig - aus welchen Gründen auch immer - abgebrochen werden oder das Lernen in anderer Form fortgesetzt wird. Die Existenz von Kursen zur Auffrischung spricht u. a. dafür, dass das organisierte Lernen einer Fremdsprache auch wieder aufgegriffen bzw. nach einer längeren Unterbrechung fortgesetzt werden kann.

Im Folgenden wird am Beispiel des Fremdsprachenlernens gezeigt, wie Erwachsene unter den Bedingungen diskontinuierlichen Lernens gleichwohl Kontinuitäten des Lebenslangen Lernens herstellen. Dies geschieht vor dem Hintergrund des Projekts „Prekäre Kontinuitäten. Der Wandel von Bildungsgestalten im großstädtischen Raum in einer Phase der forcierten Institutionalisierung des Lebenslangen Lernens“ (DFG-Gz. KA 642/4-1/2), dessen Sample aus thematisch fokussierten bildungsbiografischen Interviews mit 85 bildungsnahen Erwachsenen besteht, von denen 50 Erwachsene nach einer ersten Erhebungswelle um 1984 nach 25 Jahren ein zweites Mal interviewt wurden. Dieses Sample wurde dann weiter in die Bildungswelten „Professionalität“, „,gesellschaftliches Engagement“, „Großstadt“, „Alter“ und „Fremdsprachen“ untergliedert. ${ }^{5}$

Den Ausgangspunkt nehmen interindividuelle Wahrnehmungen der gleichen Diskontinuität am Beispiel eines Ehepaars, das in unterschiedlichen Settings gemeinsam Italienisch lernt. Die Wahrnehmungsunterschiede erweisen sich als abhängig von der jeweils konstruierten bzw. dargestellten Lerneridentität. Der Vergleich zwischen Erstund Zweitinterviews lässt erkennen, dass die unterschiedliche Wahrnehmung sich intraindividuell deutlich wandelt - zusammen mit einem weniger prononcierten, aber durchaus erkennbaren intraindividuellen Wandel der konstruierten Lerneridentität.

In einem zweiten Schritt werden zusätzliche Fälle aus dem Korpus herangezogen, die die am Beispiel der Fälle von Herrn und Frau Weber herausgearbeiteten kontinuitätsherstellenden Modi des Umgangs mit Diskontinuitäten (heroisches, gegen Widerstände sich behauptendes Lernen und selbstverständliches, mitlaufendes Lernen) bestätigen, differenzieren und um einen zusätzlichen Modus erweitern. Diese Analysen lassen erkennen, dass die individuelle und situativ bedingte (meist nachträgliche) Wahrnehmung von Diskontinuitäten generell an das Bestreben gekoppelt ist, nicht nur das eigene Lernen, sondern auch das Nicht-Lernen als kontinuierlichen Vorgang darzustellen, der der eigenen Identität als realer und potenzieller Sprachenlerner entspricht.

\section{Diskontinuierliche Lernpraktiken und Lerneridentitäten im Wandel}

Herr und Frau Weber wurden jeweils zu zwei Zeitpunkten interviewt: 1984 und 2007. Zum Zeitpunkt des ersten Interviews ist Herr Weber Anfang 40, zum Zeitpunkt des zweiten befindet er sich im Ruhestand. Frau Weber ist seit den 1970er Jahren, als ihre Kinder geboren wurden, Hausfrau. Das Paar hat sich 1980 ein Haus in Italien gebaut und lernt seitdem gemeinsam Italienisch.

${ }^{5}$ Für nähere Erläuterungen zum Projekt vgl. Fischer und Kade 2012, insbes. S. 617 ff. 


\subsection{Wahrnehmung diskontinuierlicher Lernpraktiken im Erstinterview}

Der 42jährige Herr Weber (TN 74 I) ${ }^{6}$ erzählt, dass er zum Italienischlernen ,halt zunächst mal in die Volkshochschule“ gegangen und „von Anfang an sehr skeptisch“ gewesen sei, ob er „das überhaupt so leisten könne“. Der VHS-Kurs fällt ihm ,als Anfänger" keineswegs leicht.

Ich hab allerdings von Anfang an mich entschieden, äh nun die Sprache zu lernen und auch diesen Kurs natürlich ganz selbstverständlich zu Ende zu machen.

Als dann aber der Folgekurs nicht zustande kommt,

„kam die Idee, dass wir eigentlich anhand des Buches uns alleine äh mit der italienischen Sprache befassen wollten. Und da haben wir noch zwei- aus dem Kurs zwei Mitstreiter gewonnen, ist es jede Woche, äh uns zu treffen und so Lektion um Lektion da durchzugehen, manchmal mit Schwierigkeiten, manchmal ohne" (TN 74 I:3).

Nicht ohne Stolz berichtet Herr Weber, dass er mit seiner Frau das Lehrbuch fast ganz durchgearbeitet habe und man zum Zeitpunkt des (ersten) Interviews „kurz vorm Examen“" stehe. Nach dieser Darstellung der Sprachlernpraxis führt die Erfahrung der institutionellen Diskontinuität zum selbstständigen Handeln. Herr und Frau Weber führen den Kurs privat weiter. Ihnen gelingt es auf diese Weise, das Italienisch-Lehrbuch in einem kursähnlichen Rahmen durchzuarbeiten. Der nächste Schritt von Herrn Weber ist vom Wunsch bestimmt, sich auf dieser Basis mit ihn interessierenden Inhalten zu beschäftigen. Er will diese Phase - gemeinsam mit seiner Frau - durch die Teilnahme an einem Sommerkurs einleiten.

Die organisationale Abfolge des Italienischlernens von Herrn Weber stellt sich als Wechsel vom lehrbuchbezogenen Kurs zum ebenfalls am Lehrbuch orientierten privat organisierten Lernzirkel und dann zum Ferienkurs als Eintritt in die Phase des inhaltlich bestimmten Lernens da. Die Folge $K$ (urs) - L(ernzirkel) - zukünftiger F(erienkurs) ist auch im Interview mit Frau Weber (TN 76 I) Thema. Sie erzählt zunächst, wie aus dem ursprünglichen Kurs mit 13 oder 14 Teilnehmenden ,immer mehr abgesprungen sind“, so dass man zum Schluss nur noch zu viert war:

Und dann haben wir das eben auf privater Basis weitergeführt (...) und eben so versucht (lacht leicht), ohne Lehrer das fortzusetzen, was - was wir eben da angefangen haben. (I: Ja) Und das machen wir ja jetzt nach wie vor, allerdings nur noch zu dritt (TN 76 I:1).

In der Darstellung von Frau Weber tritt die allmähliche Auflösung des ursprünglichen Kurses in den Vordergrund. Das selbstorganisierte Lernen wird als wenig spektakulär, als ein sich aus den Umständen „eben“ ergebender Versuch bezeichnet. Erst

\footnotetext{
${ }^{6}$ Der Kürzel TN bedeutet Teilnehmender, der Kürzel KL Kursleitender. Die Nummerierungen verweisen auf die Nummerierung der Interviews im Grundsample von 1984 mit 85 Teilnehmenden bzw. Kursleitenden. Die Erhebungswellen werden mit römischen Zahlen markiert; I steht für die erste Erhebungswelle von 1984, II für die zweite Erhebungswelle von 2009. Die Quell-Seiten der Zitate sind mit arabischen Ziffern bezeichnet.
} 
als die Interviewerin nachfragt, berichtet Frau Weber, dass sie sich schon vor dem Besuch des Kurses mit ihrem Mann mit dem Italienischen beschäftigt hat:

(...) vor drei Jahren praktisch haben wir angefangen. Und dann haben wir uns auch noch ein Buch gekauft mit Kassetten dazu, also vor dem, vor der Volkshochschule. Und jetzt machen wir halt nur noch mit dem - anhand von dem Buch (TN 76 I:2).

Durch diese Korrektur ergibt sich die Abfolge $S$ (elbstständiges Lernen) - K(urs) $L$ (ernzirkel) - zukünftiger $F$ (erienkurs). Der Übergang vom Kurs zum Lernzirkel wird dabei nicht als Steigerung, sondern als Reduzierung (,,halt nur noch“) dargestellt - auch wenn eine Lernbewegung der faktisch zunehmenden Emanzipation (von den Hörkassetten einerseits und von der institutionellen Strukturvorgabe andererseits) nachgezeichnet wird. Die durch die Nicht-Fortführung des VHS-Kurses bedingte Diskontinuität wird nicht als eine dramatische erfahren. Sie hat sich bereits angekündigt und wird durch die soziale Kontinuität abgemildert.

\subsection{Wahrnehmung diskontinuierlicher Lernpraktiken im Zweitinterview}

Bei der Befragung im Jahr 2007 berichtet Herr Weber (TN 74 II), er habe Anfang der 1980er Jahre ,entschieden“, „Italienisch zu lernen. Nicht aus Jux und Dollerei, sondern weil meine Familie am (Name eines Italienischen Sees) eine Wohnung gekauft hatte“. Obwohl er dort in deutscher Nachbarschaft lebte, war für ihn „klar“, dass er die Sprache lernen muss: „Ich wollte es“.

Angefangen habe er, autodidaktisch zu lernen, dann drei Monate einen Volkshochschulkurs besucht, „um die Aussprache zu lernen“. Im Anschluss habe er mehrere Lehrbücher selber durchgearbeitet und dann ,eine eigene Lehrmethode entwickelt“" und ,über diese Methode, über Jahre hinweg, sehr gut Italienisch gelernt":

Sowohl der privat organisierte Lernzirkel als auch der damals geplante Ferienkurs werden nicht erinnert bzw. nicht erwähnt. Die Abfolge der Lernpraktiken erscheint nunmehr als primär selbstständiges Lernen, das nur durch einen kurzen Kursbesuch unterbrochen wurde: $S$ (elbstständiges Lernen) - K(urs) - $S$ (elbstständiges Lernen). ${ }^{7}$ Insgesamt ist die Sprachlernpraktik von Herrn Weber (II) nunmehr durch den unbedingten Willen, die Sprache zu erlernen und ein bemerkenswert intensives und langandauerndes Lernverhalten gekennzeichnet. Von dem Interviewten selbst wird das autodidaktische Moment hervorgehoben. Es führte sogar zur Entwicklung einer eigenen (Selbst-)Lernmethode. Der Interviewte deutet an, er habe das - insgesamt erfolgreiche - Italienischlernen abgeschlossen. Die Tatsache, dass er zusammen mit seiner Frau gelernt hat, bleibt in diesem Zusammenhang unerwähnt. Herr Weber (II) stellt sich eher als ,heroischen“ Einzelkämpfer dar, ${ }^{8}$ der mit großem Einsatz trotz beein-

\footnotetext{
${ }^{7}$ Die hier verwendete Darstellung ist locker orientiert am lebenslauftheoretischen Konzept der Sequenz als Abfolge von mindestens zwei aufeinanderfolgenden Übergängen (Sackmann und Wingens 2001). Demnach würde es sich bei der Form S-K-S um den Sequenztypus ,Zwischenstatus“ handeln, der in Bezug auf die Prozesszeit als SSSS-K-SSSSSSSS... dargestellt werden könnte, (wobei die Anzahl der Buchstaben die ungefähre Dauer der Lernform bezeichnet).

${ }^{8}$ Der Begriff des „Heroischen“ fungiert als eine Art Metapher, die auf die Selbstdarstellung eines sich gegen fast übermächtige Widerstände allein durchsetzenden Kämpfers verweist (zur soziologischen Ver-
} 
trächtigender früher Lernerfahrungen im Erwachsenenalter erfolgreich eine Fremdsprache erlernt und eine dem schulischen Lehren überlegene Selbstlernmethode entwickelt hat (die er dann auch später beim Erlernen der für ihn neuen Fremdsprache Englisch anwendet). Diskontinuität wird nicht passiv erfahren, sondern von ihm selbst aktiv hergestellt. Der Erzähler ist auf seine eigene Lerngeschichte fokussiert. Er ,vergisst“ darüber das gemeinsame Lernen mit seiner Frau und das gemeinsame Lernen mit anderen in der Gruppe: Der VHS-Kurs wird von Herrn Weber nicht als soziale Situation erinnert, sondern als kurzfristig benötigtes Mittel, das zu lernen, was allein nicht möglich schien.

Frau Weber(II) erinnert sich im zweiten Interview, sie habe zusammen mit ihrem Mann „früher auch mal einen Kurs (...) von der Volkshochschule, Italienischkurs“, gemacht, und zwar „ein paar Semester“. Zum Schluss waren sie „bloß noch zu viert“. Sie beschlossen dann, den Kurs privat fortzuführen:

Ohne Lehrer. (I: Ah ja) Nee, das heißt, zuerst mit, genau, mit der Lehrerin, das war eine Lehrerin, genau. Da hatten wir das noch privat gemacht mit der Lehrerin, und dann irgendwann haben wir gesagt, nee, das können wir eigentlich jetzt auch alleine machen. Das Buch haben wir. Und dann haben wir das privat gemacht, ähm, das ging sogar eine ganze Weile ganz gut, zum Schluss waren also mein Mann und ich und, und noch eine Frau, und zum Schluss waren nur noch die Frau und ich, da ist mein Mann auch gar nicht mehr gekommen. Und irgendwann haben wir es dann ganz sausen lassen. Also dann war gar nichts mehr. (lacht) Aber es ging doch immerhin einige, einige Jahre so gut (TN 76 II:17).

Bei Frau Weber (II) ist in der Rückschau des zweiten Interviews nach fast 25 Jahren die erzählte Abfolge ihrer Lernpraktiken mithin von einem einzigen Wechsel gekennzeichnet: $K$ (urs) - $L$ (ernzirkel). Anders als bei ihrem Mann scheint für Frau Weber (II) der Kurs keine große Herausforderung darzustellen. Er knüpft an ein schulisches Lernen an. Nicht die absolvierten Lektionen und das damit erreichte Lernniveau, sondern die Zahl der besuchten Semester werden von Frau Weber erwähnt: Das Gelernte tritt hinter die Dauer des Kursbesuchs zurück. Die Tatsache, dass der Kurs von der übrig gebliebenen Gruppe allein weitergeführt wird, erscheint nicht als besondere Leistung, sondern fast als Selbstverständlichkeit. Es ist auch keine Rede davon, dass ihr Mann die Organisation übernommen und den Kurs zu einem erfolgreichen Ende geführt hat. Er wird im Gegenteil als weiterer drop-out beschrieben, dessen Fehlen zum endgültigen Ende beigetragen hat.

Im Blick auf die Situation zum Zeitpunkt des zweiten Interviews wird deutlich, dass für Frau Weber mit dem Ende des institutionell und des privat organisierten gemeinschaftlichen Lernens dieses selbst kein Ende gefunden, aber auch keine Steigerung erfahren hat:

Und, ja, und jetzt, ähm, mit Italienisch, wenn wir da unten sind, dann haben wir ja, gehen wir ja da einkaufen und, die Leute, die sprechen zwar auch teilweise Deutsch, aber wir versuchen halt Italienisch zu reden und, ja, und gut, damals, 
wie wir das Haus da gebaut haben, mussten wir ja Italienisch reden, weil kaum jemand Deutsch konnte, und gerade von den Handwerkern eigentlich... (lacht). Und das ging aber ganz gut. Und, ja, und jetzt versuchen wir halt, ab und zu mal eine Zeitung, italienische Zeitung zu lesen oder, ja, Fernsehen kriegen wir jetzt in Italien keinen, das RAI da, das kriegen wir nicht mehr. Und, ja, jetzt versuchen wir es einfach irgendwie. Also dann zwischendrin auch mal ein paar Vokabeln so zu lernen und, man vergisst es ja leider immer alles so schnell (lacht) (TN 76 II:18).

Unter Berücksichtigung der aktuellen Gegenwart erscheint die Abfolge im Fall von Frau Weber (II) quasi organisch als Weg vom curricularen Lernen zur gelegentlichen Anwendung und Erweiterung des Gelernten: $K$ (urs) - L(ernzirkel) - S(elbstständiges Lernen). Dabei stellt Frau Weber die Beschäftigung mit der italienischen Sprache als eine Lernpraktik dar, die sie mit ihrem Mann verbindet. Sie dient zwar der Bewältigung von Alltagskommunikationen und dem Medienkonsum, hat aber keineswegs zu einer Perfektion geführt. Lachend akzeptiert sie diese - für das langjährige Fremdsprachenlernen vor allem älterer Erwachsener typische - Situation, in der es eher um den Erhalt als um eine Steigerung von Kenntnissen geht.

\subsection{Herstellung von Lerneridentitäten im Erst-und Zweitinterview}

Real bestehende institutionell-organisatorische, örtliche, personelle oder lerngegenstandsbezogene Diskontinuitäten bei der Ausübung von Lernpraktiken werden - wie die knappen, eher illustrierenden Fallskizzen zeigen - individuell selektiv und unterschiedlich wahrgenommen und erinnert. Die unterschiedlichen Abfolgen scheinen dabei im Dienst des Bemühens zu stehen, dem eigenen Lernprozess eine Gestalt der Kontinuität zu geben. Herr Weber (I) zeichnet im Erstinterview eine Linie der zunehmenden Selbstbestimmung des Lernens, die sich in der Erinnerung (und unter Hinzunahme der weiteren Entwicklung) verstärkt und auf die eigene Person fokussiert. Frau Weber (I) sieht eher eine organische Entwicklung der abnehmenden Intensität des Lernens, das jeweils an unterschiedliche Personen und Situationen gebunden ist.

Diskontinuitätswahrnehmungen sind so immer mit der Konstruktion von jeweils eigenen identitätsbezogenen Kontinuitäten verbunden: hier einerseits die Identität des sich gegen Widerstände durchsetzenden Lerners, der später einst nicht erfüllte Lernanforderungen bravourös meistert, und andererseits die Identität der ,mitschwimmenden“ Lernerin, die sich unaufgeregt wechselnden Angebotsmöglichkeiten und sozialen Konstellationen anpasst.

Die Zuweisung von unterschiedlichen, fast konträren Lerneridentitäten: die des heroischen, gegen Widerstände ankämpfenden Lerners und die der unaufgeregt entsprechende Gelegenheiten ergreifenden, eher „mitschwimmenden“ Lernerin über den von den Erhebungszeitpunkten markierten gesamten Zeitraum erweist sich bei näherer Betrachtung noch als differenzierbar: So werden von Herrn Weber (II) im zweiten Interview die im ersten Interview erinnerten Selbstzweifel und Schwierigkeiten bei der Teilnahme am VHS-Sprachkurs (s. o.) nicht mehr erwähnt. Die damalige Erinnerung an die Versagensangst des fremdorganisiert Lernenden ist vom Bild des forschen, die demütigenden Erfahrungen der schulischen Vergangenheit 
bekämpfenden erfolgreichen (Selbst-)Lerners gelöscht. Auch bei Frau Weber lassen sich Akzentverschiebungen beobachten: Zwar bleibt Lernen bei ihr ein Handeln, das selbstverständlich praktischen Zwecken dient und das unabhängig von äußeren Veränderungen möglichst lange, aber nicht unbedingt gegen Widerstände beibehalten wird. Verändert hat sich im Laufe der Jahre jedoch ihre Darstellung der Gemeinsamkeit des Lernens. Im Erstinterview dominiert die soziale Kontinuität des Lernens mit ihrem Mann und mit der Lerngruppe; im zweiten Interview erinnert Frau Weber (II) sich, dass ihr Mann den Lernzirkel vor ihr verlassen und damit zu dessen Beendigung beigetragen hat. Trotzdem führt sie die Perspektive der Darstellung des gemeinsamen Italienischlernens mit ihrem Mann auch im Zweitinterview fort, nur handelt es sich jetzt nicht mehr um das heroische systematische Anfangslernen, sondern um den Versuch, eher unsystematisch (,einfach irgendwie“) dem Verfall der Sprachkenntnisse vorzubeugen bzw. Neues in dem Bewusstsein zu lernen, dass man es möglicherweise bald wieder vergessen haben wird. In der Darstellung ihres Mannes fehlt diese Phase des Lernens bzw. des Kampfes gegen das Verlernen. Herr Weber berichtet dagegen ausführlich von einer - anspruchsvollen - neuen Sportart, der er sich in der Zeit des Ruhestands widmet. Diese habe er sich - so wie einst das Italienischlernen - weitgehend selbstständig angeeignet und es dabei zu überdurchschnittlichen Erfolgen gebracht. Da er diese Sportart vorwiegend im englischsprachigen Ausland ausübt, musste er sich auch mit der für ihn im Kern neuen Fremdsprache Englisch beschäftigen. Dabei habe er auf seine beim Italienischlernen entwickelte autodidaktische Methode zurückgegriffen. Er bewahrt das Selbstbild eines heroischen Einzelkämpfers, indem er eine andere Lernpraktik (Sportart) und eine andere Fremdsprache (Englisch) wählt. Auch Frau Weber berichtet im Zweitinterview von sportlichen Aktivitäten (Gymnastikkurs), die sie aber auch schon zur Zeit des Erstinterviews ausgeübt hat. Die Teilnahme an Kursen und dann sogar auch die Kursleitung wird ihrem Selbstbild als Lernerin gemäß als eine langjährig betriebene, wenig spektakuläre, in einer Gruppe mit anderen Frauen durchaus erfolgreich ausgeübte Lernbzw. Ausübungspraktik dargestellt, die gelegentlich auch hinter anderen Aktivitäten zurückstehen muss. Diese Darstellung lässt einen selbstverständlichen, undramatischen Umgang mit Diskontinuitäten erkennen, der diese selbst eher nivelliert. Demgegenüber interpretiert Herr Weber Diskontinuitäten als Entwicklungschance. Er akzentuiert Anfänge und Abschlüsse von Lernaktivitäten und betont Unterschiede. Im Fall von Frau Weber werden Diskontinuitäten als selbstverständliche Normalität, im Fall von Herrn Weber als (,heroisch“ bewältigte) Herausforderung bzw. selbst herbeigeführte Veränderung dargestellt.

\section{Identitätsbezogene Kontinuitätsmodulierungen diskontinuierlicher Lernpraktiken}

An den Fällen Herr und Frau Weber I/II wurde ein Möglichkeitsraum entworfen, wie erwachsene Lerner mit den Diskontinuitäten des mit Kontinuitätserwartungen verbundenen Lebenslangen Lernens umgehen. Damit sind Endpunkte einer Skala bezeichnet, auf der weitere Fälle angeordnet werden können. Die Analyse zusätzlicher Fälle hat zu einer Differenzierung der beiden extremen kontinuitätswahren- 
den Lerner-Identitäten geführt und darüber hinaus dann zu einer Erweiterung des ursprünglich bipolaren Modells zu einem ternären Modell der Lernformen des Lebenslaufs.

\section{1 „Heroische“ Lerner}

Frau Falkner (TN 78), ${ }^{9}$ eine unverheiratete Chemotechnikerin, die mit ihrer Mutter bis zu deren Tod zusammenlebte, nach eigener Aussage ein „richtiger Sprachenfan“, hat zum Zeitpunkt des Erstinterviews Französisch-, Norwegisch- und SchwedischKurse besucht und sich mit Altgriechisch beschäftigt. 25 Jahre später berichtet sie, dass sie an einem Englischkurs für Senioren teilnimmt und vor einigen Jahren Bulgarisch zu lernen versucht hat.

Die Kontinuität des Lernens derartig vieler und unterschiedlicher, darunter als anspruchsvoll oder schwierig geltender Sprachen, erweist sich bei näherer Betrachtung als der Versuch, das „Trauma“ des fehlenden Abiturs zu bewältigen - eine Erfahrung, die den schulischen Misserfolgserfahrungen von Herrn Weber vergleichbar ist. Wie sie erst im zweiten Interview erzählt, hat sie stark darunter gelitten, nicht das Abitur zu haben.

Das extensive Besuchen von Kursen als Kompensation für ein vorenthaltenes Abitur prägt auch die Bildungsbiografie von Frau Mansfeld (TN 58). Die vorenthaltene schulische Bildung wird zum Motor eines dieses Bildungsdefizits übersteigenden Wissenserwerbs. Sie wird zum Anlass kontinuierlichen, diskontinuitätsindifferenten Lernens im Erwachsenenalter. Englisch hat sie in Kursen der Volkshochschule, des British Council und betriebsintern gelernt. Diese institutionelle Diskontinuität gerät in der Langzeitperspektive ebenso aus dem Blickfeld wie die Unterbrechungen des Spanischlernens, das sie im Alter beginnt.

Institutionelle, personelle und auf den Lerngegenstand bezogene Diskontinuitäten können auch dann für die Identität eines intensiven Sprachenlerners folgenlos bleiben, wenn sie einen Lernabbruch zur Folge haben. So erzählt Frau Falkner, dass sie - gegen den Widerstand ihrer Mutter, die ihre Bildungsambitionen nicht würdigt - zwei Semester lang einen anspruchsvollen Altgriechisch-Kurs bei einem Dozenten besucht hat, der später als Lehrbeauftragter an der Universität arbeitet. Als der Dozent den Kurs nicht weiterführt und sie auffordert, in einen Kurs zu wechseln, der schon sehr viel weiter ist, hat sie Schwierigkeiten, mitzukommen. Die Folge davon ist, dass sie „dann leider aufgehört“ hat. Zwar verhindert diese drohende personelle Diskontinuität (zunächst) das weitere Lernen. Aber TN 78 stellt dieses Ende nicht als endgültig dar. Der mehr oder weniger erzwungene Abbruch erscheint als bloß vorläufig, eine Wiederaufnahme des Lernens wird für möglich, ja sogar für wahrscheinlich gehalten: „Ja, ich werde irgendwann mal wieder alte Sprachen machen. Ich weiß das ganz bestimmt, weil mich das, das reizt mich immer noch“ (TN 78 I:16).

Auch ein endgütiger Abbruch des Lernens einer bestimmten Sprache muss die Kontinuität einer erfolgreichen Sprachlernbiografie nicht grundlegend infrage stellen. Wie solche Diskontinuitätserfahrungen in eine entwickelte Lerneridentität integ-

${ }^{9}$ Die im Folgenden angeführten Fälle werden unter anderen Fragestellungen behandelt in Kade und Nolda 2012a, b sowie in Kade und Nolda 2014a, b, c. 
riert werden können, macht der Fall der zunächst als Kursleiterin interviewten Frau Baumgartner (KL 15) sichtbar.

Frau Baumgartner, 1942 geboren, ist eine ausgebildete Dolmetscherin und Verlagslektorin von Sprachlehrwerken, die selbst als Spanischkursleiterin tätig ist. 2008 wird sie zum zweiten Mal interviewt - vier Jahre, nachdem sie in Rente gegangen und von der Großstadt in eine Kleinstadt gezogen ist. Im Interview erzählt sie, dass sie nach dem Umzug dort Kurse in Türkisch und Arabisch belegt hat. Für diese beiden ,seltenen“ Sprachen war sie auch im Verlag zuständig, beherrschte sie allerdings nicht. Mit diesem Lernvorhaben folgt sie dem Muster, das sie als Lektorin in Bezug auf das Spanische realisiert hat, nämlich Sprachkenntnis und Kurserfahrung mit der Lektorierung von Sprachlehrwerken zu verbinden. Auch nach der Verrentung plant KL15 noch die Herausgabe eines Arabisch-Lehrwerks mit dem Dozenten, bei dem sie in der Großstadt an dessen Sprachschule Arabisch zu lernen angefangen hatte. Nach ihrem Umzug setzt sie das Arabischlernen zunächst an ihrem neuen Wohnort fort. Sie besucht einen Arabisch-Kurs an der Volkshochschule, bricht ihn allerdings nach kurzer Zeit ab. Für den Abbruch verantwortlich macht sie die Diskontinuität von Lernort, Institution, Lehrkraft und Methode. Weil es eine als dramatisch erfahrene örtlich, institutionell, personell und didaktisch markierte Diskontinuität ist, die zum Abbruch des Lernens führt, wird von dieser Diskontinuität ihr Selbstbild als überaus engagierte Sprachenlernerin nicht berührt. Dieses basiert auf dem anspruchsvollen, lebensgeschichtlich tief verankerten Ziel einer Near-native-speaker-Kompetenz, wie es bereits hinter der frühen Entscheidung nach dem Abitur stand, statt Romanistik zu studieren eine Dolmetscherausbildung zu machen.

\subsection{Selbstverständlich-beiläufige Lerner}

Frau Baumer (TN 80 I) erzählt im Erstinterview davon, dass sie als Fünfzigjährige nach der Teilnahme an diversen Kursen der Volkshochschule, in privaten Sprachschulen und im Rahmen eines Sommerkurses nunmehr in Spanien Spanisch lernt. Die genaue Dauer der Kursteilnahme ist ihr nicht präsent. Was sie hervorhebt, ist das Spannungsverhältnis zwischen der Kontinuität der Teilnahme und den unterschiedlichen Anlässen. Im Erstinterview berichtet sie von ihrem Wunsch, nach Südamerika zu reisen und dort auch eventuell berufstätig zu sein.

Wenn man dort mal auf Reise ist, quer durch die Welt und nicht bloß in Europa, dann braucht man halt Englisch oder eben in Südamerika quer durch Spanisch (TN 80 I: 10).

Spanisch zu lernen ist für TN 80 II dabei nicht eine irgendwie interessante Herausforderung. Sie lernt Spanisch nicht wegen der Schönheit der Sprache, wie etwa TN 78 das Altgriechische, sondern für den Gebrauch in Alltagssituationen. Dabei ist die Teilnahme an Sprachkursen für Frau Baumer eine alltägliche Praktik. Entsprechend werden negative Erfahrungen mit einzelnen Kursleitenden ebenso hingenommen wie Pausen zwischen verschiedenen Kursen. Dass das Erlernen einer Fremdsprache unter unterschiedlichen, auch unterschiedlich geeigneten Bedingungen stattfindet, erscheint TN 80 II als selbstverständlich. Dies führt zur Nivellierung kursbezogener Diskontinuitäten. 
Ebenso wie bei Frau Weber spielt auch bei TN 80 der (offenbar als selbstverständlich angenommene) Lernfortschritt keine zentrale Rolle. Eher geht es bei der Kursteilnahme darum, ,dabei zu bleiben“; und dies auch dann, wenn keine unmittelbare Notwendigkeit zum Erlernen einer spezifischen Fremdsprache besteht. Rückblickend erzählt sie im zweiten Interview, nunmehr 75 Jahre alt, dass sie über 20 Jahre in Bolivien gelebt und gearbeitet hat und ihren Lebensabend in Deutschland verbringen will. An den damaligen Spanischkursen habe sie gezielt mit Blick auf eine Tätigkeit in Südamerika teilgenommen. Ungeachtet dieser im Nachhinein vielleicht etwas überakzentuierten berufsorientierten Gerichtetheit des Lernens (im ersten Interview schien das touristische Motiv zumindest gleichbedeutend zu sein), fällt die Gelassenheit auf, mit der die Interviewte dem Spanischlernen mit seinen institutionellen und personellen Diskontinuitäten, zeitlichen Unterbrechungen sowie unterschiedlich großer Lernmotivation nachgeht. Kursförmiges Lernen scheint in dieser Erzählung eher Vorbereitung auf die Sprachbeherrschung zu sein, die dann im Land selbst endgültig erwartet wird oder zumindest den dortigen Anforderungen gerecht wird. Perfektion im Sinne des (bescheinigten) Erreichens einer hohen Kompetenzstufe wird von TN 80 II nicht angestrebt.

Trotz einer lebensgeschichtlich und unter Karrieregesichtspunkten hohen Bedeutung des Sprachenlernens verhält TN 80 II sich dazu somit als einer eher beiläufig ausgeübten Praktik. Mit dieser normalisierenden Haltung ist eine Nivellierung aller Diskontinuitäten des Sprachenlernens verbunden. Das Sprachenlernen ist eine normale Lebenspraktik. Sie steht nicht im Zentrum des Lebens, sie bildet keinen Kern der Identität. Auffallend in diesem Zusammenhang ist auch, dass Frau Baumer wie auch schon Frau Weber - kaum von ihren Erfolgen spricht oder dem erreichten Niveau. Wovon sie vor allem sprechen, sind die - indes nicht als gravierend geschilderten - Probleme langjährigen Sprachenlernens wie zeitweilige Motivationsmängel bzw. altersbedingtes Vergessen.

\subsection{Potenzielle Lerner}

Im Folgenden soll ein weiterer Typus des Lebenslangen Lerners ins Blickfeld gerückt werden. Er ist bislang noch weitgehend unbeachtet geblieben; wohl nicht zuletzt deshalb, weil er nicht bereits auf einer stattgefundenen Lernpraxis aufbaut, sondern diese - Ausdruck einer entwickelten Lernbereitschaft (Nolda 2004) - eher als eine Option thematisiert wird. ${ }^{10}$

Anders als der real sich gegen Widerstände durchsetzende und dabei erfolgreiche intentionale heroische Lerner und der über Jahre hinweg mehr oder weniger kontinuierlich lernende Beiläufigkeitslerner, der davon aber wenig Aufhebens macht, wird unter den Bedingungen der gesellschaftlich durchgesetzten Norm des Lebenslangen Lernens von dem, der über einen längeren Zeitraum nicht lernt, erwartet, dass er dieses Defizit in irgendeiner Weise begründet, zumindest zu ihm Stellung nimmt; verstärkt noch einmal in bildungsbiografischen Interviews, die ja strukturell und auch interaktiv mit einer Lern- und Bildungserwartung verknüpft sind. Von zukünftigem, somit möglichem Lernen zu erzählen, ist eine - in unserem Sample nicht selten anzufindende

\footnotetext{
${ }^{10}$ In Anklang an Robert Musils Sozialfigur des Möglichkeitsmenschen (dazu auch Gross 2005) könnte man diese dritte Gestalt des Lebenslangen Lerners auch als Möglichkeitslerner bezeichnen.
} 
Strategie, vergangenes und auch gegenwärtiges Nicht-Lernen so zu legitimieren, dass dieses eine Lerneridentität nicht infrage stellt, sondern eher noch einmal bekräftigt.

Solche Begründungen gelingen am einfachsten, wenn an kurzfristiges reales Lernen im Erwachsenenalter angeschlossen werden kann. So bedauert Frau Brüning (TN 29 II), die nach der Schulzeit auf einem humanistischen Gymnasium und vor Beginn ihrer Berufstätigkeit Spanisch- und Französischkurse besucht hatte, dass sie angesichts ihres anspruchsvollen Berufs dafür zu wenig Zeit habe und prophezeit für die Zeit nach Beendigung ihrer Berufstätigkeit: „Ja, ja, da werde ich mit Sicherheit wieder verstärkt in die, in die Sprachen einsteigen“" (TN 29 II:12). Die Erzählerin scheint fest davon überzeugt zu sein, auch nach vielen Jahren bzw. Jahrzehnten wieder ,einsteigen“ zu können, wobei sie nicht nur ihr bereits bekannte, sondern auch neue Sprachen (,das Italienisch steht schon an“, TN 29 II: 7) meint.

Diese Haltung ist keineswegs nur mit dem Habitus akademisch Gebildeter verbunden. So erzählt Frau Lohse (TN 64 I), beim ersten Interview 34 Jahre alt und aktuell arbeitslos, dass sie sich im Hinblick auf einen nach der Familienphase geplanten Wiedereinstieg ins Berufsleben für einen VHS-Englischkurs angemeldet habe. Beim zweiten Interview, Frau Lohse steht mittlerweile vor der Rente, hat sich an diesem Zustand wenig geändert. Sie kann sich vage an Kursbesuche erinnern, scheint auch wenige Lernfortschritte im Englischen gemacht zu haben, nimmt aber nach wie vor für sich in Anspruch, die Sprache lernen zu können, wenn es denn notwendig sei. Diskontinuitäten in Bezug auf Institution, Lehrperson, Lehrmethode kommen dabei erst gar nicht in den Blick - die Möglichkeit aber, das Lernen zu aktualisieren, scheint über das gesamte Leben hinweg permanent zu bestehen, an der eigenen Lernfähigkeit wird nicht gezweifelt. Trotz weitgehenden Nicht-Lernens besteht Lernen als Option weiter. Es bildet gewissermaßen trotz seiner Abwesenheit ein virtuelles Kontinuum, das die faktische Diskontinuität von Lernen und Nicht-Lernen bzw. geplantem und realisiertem Lernen nicht nur nivelliert, sondern sogar ausblendet.

Vergleichbar damit ist die Einstellung der sich selbst als sprachbegabt bezeichnenden Frau Schauer (TN 39), die - inzwischen von der Großstadt an die tschechische Grenze gezogen - im zweiten Interview von der Überlegung erzählt, eventuell einen Tschechisch-Kurs zu belegen. Eine vergleichsweise ungewöhnliche Sprache zu lernen, ist für Frau Schauer nicht eine Sache der Begabung oder des Alters, sondern etwas, das - bei Bedarf - jederzeit realisiert werden kann. Die Kontinuität des potenziellen Lernens wird aufrechterhalten. Institutionelle Unterschiede haben keine Bedeutung. Probleme, die dadurch eventuell entstehen könnten, können durch den Wechsel der Lernform behoben werden.

Diese Beispiele verdeutlichen, dass die Generierung einer Lerneridentität, die an kontinuierliches Lebenslanges Lernen geknüpft ist, nicht an das nachweisbare Ausüben von Lernpraktiken gebunden ist. Nicht nur kurzfristige Unterbrechungen, sondern auch vergleichsweise lange Phasen des Nicht-Lernens oder vage Vorstellungen eines möglichen Lernens in der Zukunft müssen somit nicht dazu führen, eine Identitätskonstruktion als Lebenslanger Lerner aufzugeben. 


\section{4 „Heroisches“ Lernen - mitlaufendes Lernen - mögliches Lernen. Formen des Lernens im Lebensverlauf}

Aufgrund der Breite des Lebenslangen Lernens, das ja das informelle Lernen mit einschließt, können Abbrüche des fremdorganisierten Sprachenlernens im Erwachsenenalter eine generelle, zahlreiche andere Lernaktivitäten enthaltende Lernbewegung im Lebenslauf nicht gefährden. Trotzdem ist auffällig, wie Sprachenlerner immer wieder (und eventuell auch immer wieder anders) versuchen, die - so gut wie unumgänglichen - Diskontinuitäten ihres Lernens zugunsten der Darstellung von Kontinuität auch dann zu nivellieren, wenn faktisches Lernen kaum nachweisbar ist. Akzentuiert werden Diskontinuitäten dagegen, wenn sie - wie bei Herrn Weber und Frau Baumgartner - zum Anlass für Entscheidungen werden, die die kontinuitätssichernde Identiät im Sinne autonomer Sprachenlerner stützen, das Lernen gegen Widerstände durchsetzen. Welche Diskontinuitäten die thematisiert werden, hängt vom jeweiligen Selbstbild als Lerner ab. Gesteuert wird die Wahrnehmung und Darstellung von Diskontinuitäten durch die Kontinuität ermöglichende, weitgehend, wenn auch nicht vollständig zeitstabile Herstellung bzw. Darstellung von Identität. Sie lässt sich mit gewisser Zurückhaltung - auf drei Formen reduzieren: die Identität als heroische(r) Lerner(in), die Identität als selbstverständliche(r) Lerner(in) und die Identität als potenzielle(r) Lerner(in). Diese Formen sind nicht mit Lernertypen zu verwechseln, die das Verhalten beim Lernen beschreiben (Kolb 1984; Schrader 2008). Es handelt sich vielmehr um Muster, die die Darstellung des eigenen Lernens in der ganzen Spanne des Lebenslaufs, und zwar in durchaus unterschiedlichen Rhythmen und Kombinationen, beeinflussen. Diese Muster sind zwar an individuellen Fällen gewonnen, aber an diese nicht fest gebunden. Das Korpus, dem die Fälle entnommen sind, gibt Anlass zu der Vermutung, dass diese tendenziell stabilen (Selbst-)Darstellungsmodi als Muster des Lernens im Lebenslauf verstanden werden können, die sich auf verschiedene Lernaktivitäten und auf das Lernen über einen größeren Zeitraum beziehen und somit reale bzw. berichtete Erlebnisse von Diskontinuität in Bezug auf Lerngegenstände, Lernorte, Lernmethoden, Lernorganisationsformen überformen. Die Hinzuziehung weiterer bildungsbiografischer Erzählungen aus anderen Projekten bzw. deren Re-Analyse könnte dazu dienen, die hier empirisch gestützt vertretene Annahme zu überprüfen bzw. zu differenzieren.

Open Access Dieser Artikel unterliegt den Bedingungen der Creative Commons Attribution License. Dadurch sind die Nutzung, Verteilung und Reproduktion erlaubt, sofern der/die Originalautor/en und die Quelle angegeben sind.

\section{Literatur}

Baecker, D. (1994). Postheroisches Management. Eine Vademecum. Berlin: Merve Verlag. Behringer, F., et al. (2004). Diskontinuierliche Erwerbsbiographien. Baltmannsweiler: Schneider Verlag. Fischer, M., \& Kade, J. (2012). Qualitative Längsschnittstudien in der Erwachsenenbildungs- und Weiterbildungsbildungsforschung. In B. Schäffer \& O. Dörner (Hrsg.), Handbuch Qualitative Erwachsenen- und Weiterbildungsforschung (S. 612-625). Opladen: Verlag Barbara Budrich. 
Früchtl, J. (2004). Das unverschämte Ich. Eine Heldengeschichte der Moderne. Frankfurt a. M.: Suhrkamp Verlag.

Gross, P. (2005). Die Multioptionsgesellschaft. Frankfurt a. M.: Suhrkamp Verlag.

Grotjahn, B., Schlak, T., \& Bernd, A. (Hrsg.). (2010). Der Faktor Alter beim Spracherwerb. Themenheft der Zeitschrift für Interkulturellen Fremdsprachenunterricht 15.1.

Hof, C., Kade, J., \& Fischer M. E. (2010). Serielle Bildungsbiographien - Auf dem Weg zu einem qualitativen Bildungspanel zum Lebenslangen Lernen. Zeitschrift für Pädagogik, 3, 328-339.

Kade, J., \& Nolda, S. (2012a). (Bildungs-)Biographie und (Bildungs-)Karriere als Formen des Lebenslaufs. Zur Rekonstruktion des Wandels von Bildungsgestalten zwischen 1984 und 2009. In I. Miethe \& H. Müller (Hrsg.), Qualitative Bildungsforschung und Bildungstheorie (S. 281-308). Opladen: Verlag Barbara Budrich.

Kade, J., \& Nolda, S. (2012b). Rekursive Bildung. Neurahmungen vergangener Lernerfahrungen Erwachsener. In C. Hof, h. von H. Felden \& S. Schmidt-Lauff (Hrsg.), Erwachsenenbildung und Lernen. Dokumentation der Jahrestagung 2011 der Sektion Erwachsenenbildung der DGfE in Hamburg (S. 119-130). Baltmannsweiler: Schneider Verlag.

Kade, J., \& Nolda, S. (2014a). 1984/2009- Bildungsbiographische Gegenwarten im Wandel von Kontextkonstellationen. Zeitschrift für Pädagogik, 4, 587-605.

Kade, J., \& Nolda, S. (2014b). Individualitätsperformanz. Bildungsbiographische Anspruchsindividualitäten in sich wandelnden Kontexten. In F. von Rosenberg \& A. Geimer (Hrsg.), Bildung unter Bedingungen kultureller Pluralität (S. 125-153). Wiesbaden: Springer Verlag.

Kade, J., \& Nolda, S. (2014c). Zwischen Entscheidung und Ereignis. Okkasionelle Bildungsbiographien im Kontext des Lebenslaufs. In I. Miethe, J. Ecarius, \& A. Tervooren (Hrsg.), Bildungsentscheidungen im Lebenslauf. Perspektiven qualitativer Forschung (S. 227-254). Opladen: Verlag Barbara Budrich.

Kade, J., \& Seitter, W. (1996). Lebenslanges Lernen - Mögliche Bildungswelten. Erwachsenenbildung, Biographie und Alltag. Opladen: Leske + Budrich.

Kolb, D. A. (1984). Experiential Learning. Experience as the Source of Learning and Development. Englewood Cliffs: Prentice-Hall.

Lucius-Höhne, G., \& Deppermann, A. (2004). Rekonstruktion narrativer Identität: Ein Arbeitsbuch zur Analyse narrativer Interviews. Wiesbaden: Springer Verlag.

Nolda, S. (2004). Das Verdrängen des Lerners durch das Lernen. Zum Umgang mit Wissen in der Wissensgesellschaft. In D. M. Meister (Hrsg.), Online-Lernen und Weiterbildung (S. 29-42). Opladen: VS Verlag für Sozialwissenschaften.

Nolda, S. (2014). Fremdsprachenlernen Erwachsener als Bildungsfolge. In A. Dörpinghaus, B. Platzer, \& U. Mietzner (Hrsg.), Bildung an ihren Grenzen. Zwischen Theorie und Forschung (S. 153-165). Darmstadt: WGB.

Rausch, S. (2014). Lernen regierbar machen. Eine diskursanalytische Perspektive auf Beiträge der Europäischen Union auf Lebenslanges Lernen. Wiesbaden: Springer Verlag.

Reckwitz, A. (2003). Grundelemente einer Theorie sozialer Praktiken. Eine sozialtheoretische Perspektive. Zeitschrift für Soziologie, 4, 282-301.

Sackmann, R., \& Wingens, M. (2001). Strukturen des Lebenslaufs: Übergang - Sequenz - Verlauf. Weinheim: Juventa Verlag.

Schmenk, B. (2002). Geschlechtsspezifisches Fremdsprachenlernen? Zur Konstruktion von geschlechtstypischen Lerner- und Lernbildern in der Fremdsprachenforschung. Tübingen: Stauffenburg Verlag.

Schrader, J. (2008). Lerntypen bei Erwachsenen: Empirische Analysen zum Lernen und Lehren in der beruflichen Weiterbildung (2. akt. Aufl.). Bad Heilbrunn: Klinkhardt Verlag. 